\title{
PSEUDO-CONVERGENT SEQUENCES AND PRÜFER DOMAINS OF INTEGER-VALUED POLYNOMIALS
}

\author{
K. ALAN LOPER AND NICHOLAS J. WERNER
}

\begin{abstract}
Let $K$ be a field with rank one valuation and $V$ the valuation domain of $K$. For a subset $E$ of $V$, the ring of integer-valued polynomials on $E$ is

$$
\operatorname{Int}(E, V)=\{f \in K[x] \mid f(E) \subseteq V\} .
$$

A question of interest regarding $\operatorname{Int}(E, V)$ is: for which $E$ is Int $(E, V)$ a Prüfer domain? In this paper, we contribute a partial answer to this question. We classify exactly when $\operatorname{Int}(E, V)$ is Prüfer in the case where the elements of $E$ comprise a pseudo-convergent sequence in $V$. Our work expands on earlier results that apply when $V$ is a discrete valuation domain.
\end{abstract}

1. Introduction. Let $D$ be an integral domain (not a field) with quotient field $K$. We define the ring of integer-valued polynomials on $D$ to be

$$
\operatorname{Int}(D)=\{f(x) \in K[x] \mid f(D) \subseteq D\} .
$$

Serious work on integer-valued polynomials began in 1919 with papers by Ostrowski [12] and Pólya [13]. These papers both focused on the $D$-module structure of Int $(D)$. More recently, Int $(D)$ has been studied as a ring. It was observed by Brizolis [2] that, if $D$ is the ring of integers of an algebraic number field, then Int $(D)$ is a Prüfer domain. The question of classifying all domains $D$ such that $\operatorname{Int}(D)$ is Prüfer then became of interest. Chabert [5] and McQuillan [9] proved, independently of one another, that when $D$ is Noetherian, Int $(D)$ is Prüfer if and only if $D$ is a Dedekind domain with all residue fields finite. For a general domain $D$, the question of when Int $(D)$ is Prüfer was completely resolved in [8].

2010 AMS Mathematics subject classification. Primary 13F20, Secondary $13 \mathrm{~F} 05$.

Keywords and phrases. Integer-valued polynomial, pseudo-convergent, Prüfer domain. 2014.

Received by the editors on January 11, 2014, and in revised form on February 24, 
A construction related to that of $\operatorname{Int}(D)$ is the ring

$$
\operatorname{Int}(S, D)=\{f(x) \in K[x] \mid f(S) \subseteq D\},
$$

where $S \subseteq D$. We call this a ring of integer-valued polynomials on a subset. The classification of when $\operatorname{Int}(S, D)$ is a Prüfer domain does not follow immediately from the classification result for Int $(D)$. In fact, it seems to be significantly harder. It is easy to show that a necessary condition for Int $(S, D)$ to be a Prüfer domain is that $D$ be a Prüfer domain. McQuillan [10] proved that, if $S$ is finite, then this condition is also sufficient.

For an infinite subset $S$ of a valuation domain $V$, Cahen, Chabert and Loper [4] examined the question of when $\operatorname{Int}(S, V)$ is a Prüfer domain. Even in this special case there is no general classification result. If $V$ is one-dimensional, then clearly the corresponding valuation induces a metric on the quotient field of $V$. We can then consider the completion of $V$ with respect to this metric. We call a subset of $V$ precompact if its completion is compact. It is proven in [4] that Int $(S, V)$ is a Prüfer domain, provided $S$ is precompact, and that this condition is necessary if the valuation is discrete. The question of the necessity of the precompactness condition for a general valuation domain was left open.

In this note, we show that precompactness is not necessary in general. To do so, we recall Ostrowski's notions of pseudo-convergent sequences and pseudo-limits (both defined below). Let $V$ be a onedimensional valuation domain, and let $E=\left\{\alpha_{0}, \alpha_{1}, \alpha_{2}, \ldots\right\}$ be a sequence of elements of $V$. Then $\operatorname{Int}(E, V)$ can be a Prüfer domain if $E$ is pseudo-convergent and $V$ does not contain a pseudo-limit for the sequence. Because $E$ is not necessarily precompact in this case, our work contributes new information regarding subsets $E$ of $V$ for which $\operatorname{Int}(E, V)$ is Prüfer.

The paper is organized as follows. Section 2 reviews the definition and basic properties of pseudo-convergent sequences. Section 3 discusses the maximal spectrum of $\operatorname{Int}(E, V)$, and Sections 4 and 5 investigate the localizations of $\operatorname{Int}(E, V)$ at these maximal ideals. Our main result, Theorem 5.2, classifies, for $E$ pseudo-convergent, exactly when Int $(E, V)$ is Prüfer. We then close the paper with some examples, ultimately demonstrating (Example 5.12) that the precompactness of $E$ is not necessary for $\operatorname{Int}(E, V)$ to be Prüfer. 
2. Pseudo-convergent sequences. Throughout, $K$ denotes a field with rank one valuation $v$, the ring

$$
V=\{a \in K \mid v(a) \geq 0\}
$$

is the valuation domain of $K$, and

$$
\mathfrak{m}=\{a \in K \mid v(a)>0\}
$$

is the maximal ideal of $V$. A sequence $\left(a_{i}\right)_{i \in \mathbb{N}}$ of elements of $K$ is called pseudo-convergent if, for all $i>j>k$, we have $v\left(a_{i}-a_{j}\right)>v\left(a_{j}-a_{k}\right)$. An element $a \in K$ is a pseudo-limit of the pseudo-convergent sequence $\left(a_{i}\right)_{i \in \mathbb{N}}$ if, for all $i>j$, we have $v\left(a-a_{i}\right)>v\left(a-a_{j}\right)$.

We let

$$
E=\left\{\alpha_{0}, \alpha_{1}, \alpha_{2}, \ldots\right\} \subseteq V
$$

be such that the sequence $\left(\alpha_{i}\right)_{i \in \mathbb{N}}$ is pseudo-convergent. We associate $E$ with the sequence $\left(\alpha_{i}\right)_{i \in \mathbb{N}}$ so that terminology for pseudo-convergent sequences carries over to $E$ (e.g., we may say that $E$ is pseudoconvergent). Given a rational function $\phi \in K(x)$, we let $v_{i}(\phi)=$ $v\left(\phi\left(\alpha_{i}\right)\right)$ for each $i \in \mathbb{N}$.

Our first lemma lists some fundamental properties of pseudoconvergent sequences. These properties will be used frequently throughout this paper.

Lemma 2.1. Let $\left(a_{i}\right)_{i \in \mathbb{N}}$ be a pseudo-convergent sequence in $K$, and let $f \in K[x]$.

(i) [7, Lemma 1]. Either

(a) $v\left(a_{i}\right)>v\left(a_{j}\right)$, for all $i>j$, or

(b) there exists $n \in \mathbb{N}$ such that $v\left(a_{i}\right)=v\left(a_{n}\right)$ for all $i \geq n$.

(ii) $\left[7\right.$, Lemma 2]. For all $i>j$, we have $v\left(a_{i}-a_{j}\right)=v\left(a_{j+1}-a_{j}\right)$.

(iii) $\left[\mathbf{1 1}\right.$, page 371]. The sequence $\left(f\left(a_{i}\right)\right)_{i \in \mathbb{N}}$ is eventually pseudoconvergent, that is, there exists $n \in \mathbb{N}$ such that, whenever $i>j>k \geq n, v\left(f\left(a_{i}\right)-f\left(a_{j}\right)\right)>v\left(f\left(a_{j}\right)-f\left(a_{k}\right)\right)$. Consequently, either

(a) $v\left(f\left(a_{i}\right)\right)>v\left(f\left(a_{j}\right)\right)$ for all $i>j \geq n$, or

(b) there exists $j^{\prime} \in \mathbb{N}, j^{\prime} \geq n$, such that $v\left(f\left(a_{i}\right)\right)=v\left(f\left(a_{j^{\prime}}\right)\right)$ for all $i \geq j^{\prime}$. 
The conditions in parts (i) and (ii) of Lemma 2.1 are important enough to warrant their own terminology.

Definition 2.2. Let $\left(a_{i}\right)_{i \in \mathbb{N}}$ be a pseudo-convergent sequence in $K$. If $v\left(a_{i}\right)>v\left(a_{j}\right)$ for all $i>j$, then we say that $\left(a_{i}\right)_{i \in \mathbb{N}}$ is increasing. If there exists $n \in \mathbb{N}$ such that $v\left(a_{i}\right)=v\left(a_{n}\right)$ for all $i \geq n$, then we say that $\left(a_{i}\right)_{i \in \mathbb{N}}$ stabilizes or is stable.

Let $f \in K[x]$, and let $n$ be as in Lemma 2.1 (iii). If $v\left(f\left(a_{i}\right)\right)>$ $v\left(f\left(a_{j}\right)\right)$ for all $i>j \geq n$, then we say that $\left(f\left(a_{i}\right)\right)_{i \in \mathbb{N}}$ is eventually increasing. If there exists $j^{\prime} \in \mathbb{N}, j^{\prime} \geq n$, such that $v\left(f\left(a_{i}\right)\right)=v\left(f\left(a_{j^{\prime}}\right)\right)$ for all $i \geq j^{\prime}$, then we say that $\left(f\left(a_{i}\right)\right)_{i \in \mathbb{N}}$ eventually stabilizes or is eventually stable.

3. The maximal spectrum of $\operatorname{Int}(E, V)$. When $S \subseteq V$, the ring Int $(S, V)$ of integer-valued polynomials on $S$ is

$$
\operatorname{Int}(S, V)=\{f \in K[x] \mid f(S) \subseteq V\} .
$$

Our focus will be on the ring Int $(E, V)$, where $E$ comprises a pseudoconvergent sequence as in Section 2. The major question we investigate is: when is Int $(E, V)$ a Prüfer domain? We will completely answer this question (Theorem 5.2) and give necessary and sufficient conditions in terms of $E$ for Int $(E, V)$ to be Prüfer.

One of the many equivalent conditions for a commutative domain $D$ to be Prüfer is that the localization of $D$ at each maximal ideal is a valuation domain (see [6, Theorem 22.1]). We will use this characterization of Prüfer domains in our work with $\operatorname{Int}(E, V)$. Hence, we require a description of all the maximal ideals of $\operatorname{Int}(E, V)$. This is the goal of the present section. The complete classification of the maximal spectrum of $\operatorname{Int}(E, V)$ is given in Corollary 3.9.

The maximal ideals of $\operatorname{Int}(E, V)$ come in two types: unitary and non-unitary. An ideal $\mathfrak{I}$ of $\operatorname{Int}(E, V)$ is unitary if $\mathfrak{I} \cap V \neq(0)$ and is non-unitary if $\mathfrak{I} \cap V=(0)$. When $\mathfrak{M}$ is a maximal ideal of $\operatorname{Int}(E, V)$, $\mathfrak{M} \cap V$ is a prime ideal of $V$ and, since we are assuming that $V$ is one-dimensional, $\mathfrak{M}$ being unitary is equivalent to having $\mathfrak{M} \cap V=\mathfrak{m}$.

When $\mathfrak{M}$ is non-unitary, we can use established theory to prove that $\operatorname{Int}(E, V)_{\mathfrak{M}}$ is a valuation domain. 
Theorem 3.1. The nonzero non-unitary prime ideals of $\operatorname{Int}(E, V)$ are in one-to-one correspondence with the irreducible polynomials of $K[x]$. To each irreducible $q \in K[x]$, we associate the prime ideal

$$
\mathfrak{P}_{q}:=q(x) K[x] \cap \operatorname{Int}(E, V),
$$

and every non-unitary prime ideal of $\operatorname{Int}(E, V)$ has this form. Moreover, the localization of $\operatorname{Int}(E, V)$ at a non-unitary maximal ideal is a valuation domain.

Proof. The characterization of the non-unitary prime ideals follows from [3, Proposition V.1.1] and the comment preceding it. For the statement about the localization, note first that $\operatorname{Int}(E, V)$ contains Int $(V)$. Let $\mathfrak{M}$ be a non-unitary maximal ideal of $\operatorname{Int}(E, V)$, and let $\mathfrak{P}=\mathfrak{M} \cap \operatorname{Int}(V)$; then, $\mathfrak{P}$ is a nonzero non-unitary prime of $\operatorname{Int}(V)$. By [3, Corollary V.1.2], Int $(V)_{\mathfrak{P}}$ is a valuation domain, and it is easy to see that $\operatorname{Int}(V)_{\mathfrak{P}}$ is contained in $\operatorname{Int}(E, V)_{\mathfrak{M}}$. Hence, $\operatorname{Int}(E, V)_{\mathfrak{M}}$ must also be a valuation domain.

In light of Theorem 3.1, we can concentrate on the unitary maximal ideals of Int $(E, V)$. This will remain our focus for the remainder of the paper.

Definition 3.2. For each $i \in \mathbb{N}$, let

$$
\mathfrak{M}_{i}=\left\{f \in \operatorname{Int}(E, V) \mid f\left(\alpha_{i}\right) \in \mathfrak{m}\right\}
$$

We also let

$$
\mathfrak{M}_{\infty}=\left\{f \in \operatorname{Int}(E, V) \mid f\left(\alpha_{i}\right) \in \mathfrak{m} \text { for all but finitely many } i \in \mathbb{N}\right\}
$$

It is easy to see that $\mathfrak{M}_{i}$ is an ideal for each $i$, and that each $\mathfrak{M}_{i}$ is distinct. Moreover, the $\mathfrak{M}_{i}$ are all maximal because $\operatorname{Int}(E, V) / \mathfrak{M}_{i} \cong$ $V / \mathfrak{m}$ via the map $f \mapsto f\left(\alpha_{i}\right) \bmod \mathfrak{m}$. The set $\mathfrak{M}_{\infty}$ is easily seen to be a prime ideal, but it is non-trivial to verify that it is maximal. For now, we can at least say that $\mathfrak{M}_{\infty}$ is distinct from all the $\mathfrak{M}_{i}$. 
Lemma 3.3. For each $i \in \mathbb{N}$, let

$$
H_{i}(x)=\left[\prod_{\substack{0 \leq \ell \leq i+1 \\ \ell \neq i}}\left(x-\alpha_{\ell}\right)\right] /\left[\prod_{\substack{0 \leq \ell \leq i+1 \\ \ell \neq i}}\left(\alpha_{i}-\alpha_{\ell}\right)\right] .
$$

Then, $H_{i} \in \operatorname{Int}(E, V)$ and has the following properties:

(i) $v_{j}\left(H_{i}\right)=\infty$ for $0 \leq j \leq i-1$ and $j=i+1$,

(ii) $v_{i}\left(H_{i}\right)=0$,

(iii) there exists $\rho>0$ such that $v_{j}\left(H_{i}\right)=\rho$ for all $j>i+1$.

Consequently, each $H_{i} \in \mathfrak{M}_{\infty} \backslash \mathfrak{M}_{i}$, and so $\mathfrak{M}_{\infty} \nsubseteq \mathbb{\mathfrak { M } _ { i }}$.

Proof. Properties (i) and (ii) are clear, since $H_{i}\left(\alpha_{j}\right)=0$ for the values of $j$ specified in (i), and $H_{i}\left(\alpha_{i}\right)=1$.

For (iii), let $\rho=v_{i+2}\left(H_{i}\right)$. Then $\rho>0$ because, for each $0 \leq \ell \leq i-1$, Lemma 2.1 says that $v\left(\alpha_{i+2}-\alpha_{\ell}\right)=v\left(\alpha_{i}-\alpha_{\ell}\right)$, and $v\left(\alpha_{i+2}-\alpha_{i+1}\right)>$ $v\left(\alpha_{i+1}-\alpha_{i}\right)$ because $E$ is pseudo-convergent. Finally, when $j>i+1$, another appeal to Lemma 2.1 gives $v\left(\alpha_{j}-\alpha_{\ell}\right)=v\left(\alpha_{i+2}-\alpha_{\ell}\right)$ for $0 \leq \ell \leq i-1$ and $\ell=i+1$, so $v_{j}\left(H_{i}\right)=\rho$. The fact that $H_{i} \in \mathfrak{M}_{\infty} \backslash \mathfrak{M}_{i}$ now follows.

In Theorem 3.8 below, we will prove that $\mathfrak{M}_{\infty}$ is maximal and that the $\mathfrak{M}_{i}$ and $\mathfrak{M}_{\infty}$ comprise the full set of unitary maximal ideals of Int $(E, V)$. Proving Theorem 3.8 requires several lemmas. In what follows, we say that $f \in \operatorname{Int}(E, V)$ is unit-valued on $E$ if $f\left(\alpha_{j}\right) \in V^{\times}$, for each $j \in \mathbb{N}$; equivalently, $v_{j}(f)=0$ for each $j$.

Lemma 3.4. Let $\mathfrak{I}$ be an ideal of $\operatorname{Int}(E, V)$ such that $\mathfrak{I} \nsubseteq \mathfrak{M}_{\infty}$ and $\mathfrak{I} \nsubseteq \mathfrak{M}_{i}$ for all $i \in \mathbb{N}$. Then, $\mathfrak{I}$ contains a polynomial that is unit-valued on $E$.

Proof. Since $\mathfrak{I} \nsubseteq \mathfrak{M}_{\infty}$, there exists $f \in \mathfrak{I}$ such that $v_{j}(f)=0$ for infinitely many $j \in \mathbb{N}$. By Lemma $2.1,\left(v_{j}(f)\right)_{j \in \mathbb{N}}$ either eventually increases or is eventually stable. Since infinitely many $v_{j}(f)$ are 0 , $\left(v_{j}(f)\right)_{j \in \mathbb{N}}$ must stabilize at 0 . Thus, there exists $n \in \mathbb{N}$ such that $v_{j}(f)=0$ for all $j \geq n$. 
We would be finished if $v_{j}(f)=0$ for $0 \leq j \leq n-1$, but this need not occur in general. However, we can use $f$ to produce another polynomial that is definitely unit-valued on $E$.

First, for $0 \leq i \leq n-1$, define

$$
G_{i}(x)=\left[\prod_{\substack{0 \leq \ell \leq n \\ \ell \neq i}}\left(x-\alpha_{\ell}\right)\right] /\left[\prod_{\substack{0 \leq \ell \leq n \\ \ell \neq i}}\left(\alpha_{i}-\alpha_{\ell}\right)\right] .
$$

Then, $v_{j}\left(G_{i}\right)=\infty$ for $0 \leq j \leq n, j \neq i$, and $v_{i}\left(G_{i}\right)=0$. By Lemma 2.1 (ii), $v_{j}\left(G_{i}\right)=v_{n+1}\left(G_{i}\right)$ for all $j \geq n+1$, and $v_{n+1}\left(G_{i}\right)>0$, because

$$
v\left(\alpha_{n+1}-\alpha_{\ell}\right)= \begin{cases}v\left(\alpha_{i}-\alpha_{\ell}\right) & 0 \leq \ell<i \\ v\left(\alpha_{\ell+1}-\alpha_{\ell}\right) & \ell>i\end{cases}
$$

and $v\left(\alpha_{\ell+1}-\alpha_{\ell}\right)>v\left(\alpha_{i}-\alpha_{\ell}\right)$ when $\ell>i$. Hence, each $G_{i} \in \operatorname{Int}(E, V)$.

Next, for each $i \in \mathbb{N}$, let $f_{i} \in \mathfrak{I} \backslash \mathfrak{M}_{i}$. Let

$$
S=\left\{0 \leq s \leq n-1 \mid v_{s}(f)>0\right\},
$$

and let

$$
F=f+\sum_{s \in S} f_{s} G_{s}
$$

Then, $F \in \mathfrak{I}$, and we claim that $F$ is unit-valued on $E$. Indeed, if $v_{j}(f)=0$, then $j \notin S$, so for each $s \in S$ we have $v_{j}\left(f_{s} G_{s}\right) \geq v_{j}\left(G_{s}\right)>0$; it follows that $v_{j}(F)=0$. On the other hand, if $v_{j}(f)>0$, then $j \in S$, so $v_{j}\left(f_{j} G_{j}\right)=0$ while $v_{j}\left(f_{s} G_{s}\right)=\infty$ for $s \neq j$. Hence, $v_{j}(F)=0$ in this case as well, and we conclude that $F$ is unit-valued on $E$.

Lemma 3.5. Let $f \in \operatorname{Int}(E, V)$. Then, the set $f(E) \bmod \mathfrak{m}$ is finite.

Proof. Since $f \in \operatorname{Int}(E, V)$, we have $v\left(f\left(\alpha_{i}\right)-f\left(\alpha_{j}\right)\right) \geq 0$ for any choice of $i$ and $j$. By Lemma 2.1 (iii), $\left(f\left(\alpha_{i}\right)\right)_{i \in \mathbb{N}}$ is eventually pseudo-convergent. Hence, after a certain point, $v\left(f\left(\alpha_{i}\right)-f\left(\alpha_{j}\right)\right)>$ $v\left(f\left(\alpha_{j}\right)-f\left(\alpha_{k}\right)\right) \geq 0$ whenever $i>j>k$. In other words, eventually $f\left(\alpha_{i}\right)-f\left(\alpha_{j}\right) \in \mathfrak{m}$ whenever $i>j$. So, the values of $f$ on $E$, reduced modulo $\mathfrak{m}$, eventually stabilize. Consequently, $f(E) \bmod \mathfrak{m}$ is finite. 
Lemma 3.6. Assume $f \in \operatorname{Int}(E, V)$ is such that $v_{j}(f)>0$ for all $j \in \mathbb{N}$. Then, $f$ is in every ideal of $\operatorname{Int}(E, V)$ above $\mathfrak{m}$ and, since $V$ is one-dimensional, $f$ is in every unitary prime ideal of $\operatorname{Int}(E, V)$.

Proof. Since each $v_{j}(f)>0$ and the sequence $\left(v_{j}(f)\right)_{j \in \mathbb{N}}$ is either eventually increasing or eventually stable, $\left(v_{j}(f)\right)_{j \in \mathbb{N}}$ attains a minimum value. Let $\beta \in V$ be such that $v_{j}(f) \geq v(\beta)>0$ for all $j \in \mathbb{N}$. Then, $f(x) / \beta \in \operatorname{Int}(E, V)$ and, since $\beta \in \mathfrak{m}$,

$$
f(x)=(f(x) / \beta) \beta
$$

is in each ideal of $\operatorname{Int}(E, V)$ containing $\mathfrak{m}$.

Proposition 3.7. Let $\mathfrak{P}$ be a unitary prime ideal of $\operatorname{Int}(E, V)$. Then, either $\mathfrak{P} \subseteq \mathfrak{M}_{\infty}$ or $\mathfrak{P} \subseteq \mathfrak{M}_{i}$ for some $i \in \mathbb{N}$.

Proof. Suppose that $\mathfrak{P} \nsubseteq \mathfrak{M}_{\infty}$ and $\mathfrak{P} \nsubseteq \mathfrak{M}_{i}$ for all $i \in \mathbb{N}$. Then, by Lemma $3.4, \mathfrak{P}$ contains a polynomial $F$ that is unit-valued on $E$. By Lemma 3.5, we can find finitely many units $u_{1}, u_{2}, \ldots, u_{t} \in V^{\times}$to represent all the residues in $F(E) \bmod \mathfrak{m}$.

Let

$$
f=\left(F-u_{1}\right)\left(F-u_{2}\right) \cdots\left(F-u_{t}\right) .
$$

Then, $f\left(\alpha_{j}\right) \in \mathfrak{m}$ for all $j \in \mathbb{N}$. Since $\mathfrak{P} \cap V=\mathfrak{m}, f \in \mathfrak{P}$ by Lemma 3.6. But $\mathfrak{P}$ is prime, so $F-u_{\ell} \in \mathfrak{P}$ for some $1 \leq \ell \leq t$, implying that $u_{\ell} \in \mathfrak{P}$. Consequently, $\mathfrak{P}=\operatorname{Int}(E, V)$, which is a contradiction.

Theorem 3.8. $\mathfrak{M}_{\infty}$ is a maximal ideal of $\operatorname{Int}(E, V)$, and the unitary maximal ideals of Int $(E, V)$ are exactly $\mathfrak{M}_{\infty}$ and $\mathfrak{M}_{i}$, for $i \in \mathbb{N}$.

Proof. Let $\mathfrak{M}$ be a maximal ideal of $\operatorname{Int}(E, V)$ containing $\mathfrak{M}_{\infty}$. Then, $\mathfrak{M}$ is unitary and, by Lemma $3.3, \mathfrak{M} \neq \mathfrak{M}_{i}$ for all $i \in \mathbb{N}$. By Proposition 3.7, we must have $\mathfrak{M}=\mathfrak{M}_{\infty}$, so $\mathfrak{M}_{\infty}$ is maximal. As Proposition 3.7 precludes the existence of unitary maximal ideals other than $\mathfrak{M}_{\infty}$ and the $\mathfrak{M}_{i}$, the theorem is proved.

We now have a complete description of the maximal spectrum of $\operatorname{Int}(E, V)$. 


\section{Corollary 3.9.}

(i) The non-unitary maximal ideals of $\operatorname{Int}(E, V)$ all have the form $q(x) K[x] \cap \operatorname{Int}(E, V)$ for some monic irreducible $q \in K[x]$.

(ii) The unitary maximal ideals of $\operatorname{Int}(E, V)$ are precisely $\mathfrak{M}_{\infty}$ and $\mathfrak{M}_{i}$, for $i \in \mathbb{N}$.

Having classified all the maximal ideals of $\operatorname{Int}(E, V)$, we next determine when the localization of $\operatorname{Int}(E, V)$ at a maximal ideal is a valuation domain. By Theorem 3.1, Int $(E, V)_{\mathfrak{M}}$ is a valuation domain for any non-unitary maximal ideal $\mathfrak{M}$. In Sections 4 and 5 , we will consider localizations at $\mathfrak{M}_{i}$ and $\mathfrak{M}_{\infty}$. As we shall see (Corollary 4.4), $\operatorname{Int}(E, V)_{\mathfrak{M}_{i}}$ is always a valuation domain. Thus, the determining factor in whether Int $(E, V)$ is Prüfer comes from the maximal ideal $\mathfrak{M}_{\infty}$.

4. Localizations at $\mathfrak{M}_{i}$. Our goal in this section is to prove that Int $(E, V)_{\mathfrak{M}_{i}}$ is a valuation domain for each $i \in \mathbb{N}$. In fact, we will prove that Int $(E, V)_{\mathfrak{M}_{i}}$ equals the valuation domain given in the following definition.

Definition 4.1. For each $i \in \mathbb{N}$, define

$$
V_{i}=\left\{\phi \in K(x) \mid \phi\left(\alpha_{i}\right) \in V\right\}=\left\{\phi \mid v_{i}(\phi) \geq 0\right\} .
$$

Lemma 4.2. For each $i \in \mathbb{N}, V_{i}$ is a valuation domain, and $\operatorname{Int}(E, V)_{\mathfrak{M}_{i}} \subseteq V_{i}$.

Proof. The set $V_{i}$ is clearly a subring of $K(x)$ and, for each $\phi \in K(x)$, either $v_{i}(\phi) \geq 0$ or $v_{i}(1 / \phi) \geq 0$. Thus, for each $\phi \in K(x)$, either $\phi \in V_{i}$ or $\phi^{-1} \in V_{i}$. By [6, Theorem 16.3], $V_{i}$ is a valuation domain. Also, $\operatorname{Int}(E, V)_{\mathfrak{M}_{i}} \subseteq V_{i}$ because, if $f \in \operatorname{Int}(E, V)$ and $g \notin \mathfrak{M}_{i}$, then

$$
v_{i}(f / g)=v_{i}(f)-v_{i}(g)=v_{i}(f)-0 \geq 0 .
$$

To show that Int $(E, V)_{\mathfrak{M}_{i}}$ equals $V_{i}$, it will suffice to demonstrate that

$$
V_{i} \subseteq \operatorname{Int}(E, V)_{\mathfrak{M}_{i}}
$$

We prove this in the next theorem by utilizing the polynomials $H_{i}$ defined in Lemma 3.3. 
Theorem 4.3. Let $i \in \mathbb{N}$. Then, $V_{i} \subseteq \operatorname{Int}(E, V)_{\mathfrak{M}_{i}}$.

Proof. Let $\phi \in V_{i}$, and write $\phi=f / g$, where $f, g \in V[x]$ with no common factors. Then, $f, g \in \operatorname{Int}(E, V)$. If $v_{i}(g)=0$, then $g \notin \mathfrak{M}_{i}$, and hence, $\phi \in \operatorname{Int}(E, V)_{\mathfrak{M}_{i}}$. So, assume that $v_{i}(g)>0$.

Let $\beta=g\left(\alpha_{i}\right)$. Then, $\beta \neq 0$ because $\phi \in V_{i}$. Let $H_{i}$ be as in Lemma 3.3. By construction, there exists $\rho>0$ such that

$$
v_{j}\left(H_{i}\right) \geq \rho \text { for } j \neq i .
$$

Since the value group of $K$ has rank one, we can choose $n \in \mathbb{N}$ such that $v_{j}\left(H_{i}^{n}\right)>v(\beta)$ for all $j \neq i$. Decompose $\phi$ as follows:

$$
\phi=\left(\left(f H_{i}^{n}\right) / \beta\right) /\left(\left(g H_{i}^{n}\right) / \beta\right) .
$$

To show that $\phi \in \operatorname{Int}(E, V)_{\mathfrak{M}_{i}}$, it suffices to show that $f H_{i}^{n} / \beta \in$ $\operatorname{Int}(E, V)$ and $g H_{i}^{n} / \beta \in \operatorname{Int}(E, V) \backslash \mathfrak{M}_{i}$.

When $j \neq i$,

$$
v_{j}\left(f H_{i}^{n} / \beta\right)=v_{j}(f)+v_{j}\left(H_{i}^{n}\right)-v(\beta),
$$

and this is non-negative because $f \in V[x]$ and

$$
v_{j}\left(H_{i}^{n}\right)>v(\beta) .
$$

Furthermore,

$$
v_{i}\left(f H_{i}^{n} / \beta\right)=v_{i}(\phi) \geq 0,
$$

because $v_{i}\left(H_{i}\right)=0$ and $\phi \in V_{i}$. So, $f H_{i}^{n} / \beta$ is a polynomial in $K[x]$ and $v_{j}\left(f H_{i}^{n} / \beta\right) \geq 0$ for all $j \in \mathbb{N}$. Hence,

$$
f H_{i}^{n} / \beta \in \operatorname{Int}(E, V) .
$$

Applying a similar argument to $g H_{i}^{n} / \beta$ shows that $v_{j}\left(g H_{i}^{n} / \beta\right) \geq 0$ for $j \neq i$ and $v_{i}\left(g H_{i}^{n} / \beta\right)=0$. Thus,

$$
g H_{i}^{n} / \beta \in \operatorname{Int}(E, V) \backslash \mathfrak{M}_{i} .
$$

Corollary 4.4. For each $i \in \mathbb{N}$, Int $(E, V)_{\mathfrak{M}_{i}}$ is a valuation domain.

Given Theorem 3.1 and Corollary 4.4, we see that Int $(E, V)$ being Prüfer depends entirely on the localization at $\mathfrak{M}_{\infty}$. 
Corollary 4.5. Int $(E, V)$ is a Prüfer domain if and only if the localization $\operatorname{Int}(E, V)_{\mathfrak{M}_{\infty}}$ is a valuation domain.

The examination of $\operatorname{Int}(E, V)_{\mathfrak{M}_{\infty}}$ is the topic of the next section.

5. Localization at $\mathfrak{M}_{\infty}$. In contrast to the situation with $\mathfrak{M}_{i}$, Int $(E, V)_{\mathfrak{M}_{\infty}}$ is not always a valuation domain; it depends on $E$. We borrow the following definitions from Kaplansky [7].

Definition 5.1. The pseudo-convergent sequence $E=\left(\alpha_{i}\right)_{i \in \mathbb{N}}$ is said to be of transcendental type if $\left(v_{j}(f)\right)_{j \in \mathbb{N}}$ eventually stabilizes for every $f \in K[x]$. If $\left(v_{j}(f)\right)_{j \in \mathbb{N}}$ is eventually strictly increasing for at least one $f \in K[x]$, then we say that $E$ is of algebraic type.

The breadth of $E$, denoted by $\operatorname{Br}(E)$, is defined to be

$$
\operatorname{Br}(E)=\left\{b \in V \mid v(b)>v\left(\alpha_{i+1}-\alpha_{i}\right) \text { for all } i \in \mathbb{N}\right\} .
$$

The breadth of $E$ always forms an ideal of $V$. Given a pseudo-limit $\alpha$ of $E$ in $K$, all other pseudo-limits of $E$ in $K$ have the form $\alpha+b$ for some $b \in \operatorname{Br}(E)$ [7, Lemma 3]. In particular, if a pseudo-limit of $E$ exists and $\operatorname{Br}(E)=(0)$, then the pseudo-limit is unique.

We can use the breadth and the type of $E$ to classify exactly when $\operatorname{Int}(E, V)_{\mathfrak{M}_{\infty}}$ is a valuation domain.

Theorem 5.2. Let $E$ be a pseudo-convergent sequence in $V$. Then, $\operatorname{Int}(E, V)_{\mathfrak{M}_{\infty}}$ is a valuation domain if and only if $E$ is of transcendental type or $\operatorname{Br}(E)=(0)$. Consequently, Int $(E, V)$ is a Prüfer domain if and only if $E$ is of transcendental type or $\operatorname{Br}(E)=(0)$.

The proof of Theorem 5.2 is more complicated than our work in earlier sections and relies on some theorems from [4]. We will prove the theorem via a number of intermediary results. We begin with the following lemma about the values of rational functions in $\operatorname{Int}(E, V)_{\mathfrak{M}_{\infty}}$.

\section{Lemma 5.3.}

(i) If $g \in \operatorname{Int}(E, V) \backslash \mathfrak{M}_{\infty}$, then $v_{j}(g)=0$ for all sufficiently large $j$. 
(ii) If $f / g \in \operatorname{Int}(E, V)_{\mathfrak{M}_{\infty}}$, then $v_{j}(f / g) \geq 0$ for all sufficiently large $j$.

Proof.

(i) Since

$$
g \in \operatorname{Int}(E, V), \quad v_{j}(g) \geq 0 \text { for all } j \in \mathbb{N} .
$$

But, since $g \notin \mathfrak{M}_{\infty}, v_{j}(g)=0$ for infinitely many $j$. So, $\left(v_{j}(g)\right)_{j \in \mathbb{N}}$ eventually stabilizes at 0 .

(ii) With $f \in \operatorname{Int}(E, V)$ and $g \in \operatorname{Int}(E, V) \backslash \mathfrak{M}_{\infty}$, we have $v_{j}(f) \geq 0$ for all $j$, and $v_{j}(g)=0$ for sufficiently large $j$. Hence, $v_{j}(f / g)$ is eventually non-negative.

Next, we will show that, if $E$ is of transcendental type, then $\operatorname{Int}(E, V)_{\mathfrak{M}_{\infty}}$ is a valuation domain. Our approach is similar to our work in Section 4.

Definition 5.4. We define

$$
V_{\infty}=\left\{\phi \in K(x) \mid \phi\left(\alpha_{i}\right) \in V \text { for all but finitely many } i \in \mathbb{N}\right\} .
$$

It is straightforward to prove that $V_{\infty}$ is a subring of $K(x)$. Whether it is a valuation domain depends on $E$.

Proposition 5.5. Assume that $E$ is of transcendental type. Then, $V_{\infty}$ is a valuation domain.

Proof. Let $\phi \in K(x)$. Since $E$ is of transcendental type, both the numerator and denominator of $\phi$ are eventually stable; hence, $\left(v_{j}(\phi)\right)_{j \in \mathbb{N}}$ also eventually stabilizes, say at $\varepsilon$. If $\varepsilon \geq 0$, then $\phi \in V_{\infty}$ and, if $\varepsilon<0$, then $1 / \phi \in V_{\infty}$.

Theorem 5.6. Assume that $E$ is of transcendental type. Then,

$$
\operatorname{Int}(E, V)_{\mathfrak{M}_{\infty}}=V_{\infty} .
$$

Proof. The containment Int $(E, V)_{\mathfrak{M}_{\infty}} \subseteq V_{\infty}$ follows from Lemma 5.3 (ii). So, it suffices to prove that $V_{\infty} \subseteq \operatorname{Int}(E, V)_{\mathfrak{M}_{\infty}}$. Let $f / g \in V_{\infty}$, where $f, g \in V[x]$, and find $n \in \mathbb{N}$ such that $v_{j}(f)$ and $v_{j}(g)$ are stable 
for all $j \geq n$. Then, $v_{j}(f / g)$ is also stable for $j \geq n$. Since $f / g \in V_{\infty}$, we must have $v_{j}(f / g) \geq 0$ for such $j$, and hence $v_{j}(f)-v_{j}(g) \geq 0$.

Let

$$
H(x)=\prod_{0 \leq \ell \leq n}\left(x-\alpha_{\ell}\right),
$$

and let

$$
\beta=g\left(\alpha_{n+1}\right) H\left(\alpha_{n+1}\right) ;
$$

note that $v(\beta)=v_{n+1}(g)+v_{n+1}(H)$. Decompose $f / g$ as

$$
f / g=(f H / \beta) /(g H / \beta) .
$$

We claim that

$$
f H / \beta \in \operatorname{Int}(E, V)
$$

and

$$
g H / \beta \in \operatorname{Int}(E, V) \backslash \mathfrak{M}_{\infty}
$$

By Lemma 2.1 (ii),

$$
v_{j}(H)=v_{n+1}(H) \text { for all } j \geq n+1 .
$$

From this, we get that

$$
v_{j}(g H / \beta)=0 \quad \text { for all } j \geq n+1,
$$

and clearly, $v_{j}(g H / \beta)=\infty$ when $0 \leq j \leq n$. So,

$$
g H / \beta \in \operatorname{Int}(E, V) \backslash \mathfrak{M}_{\infty} .
$$

Finally, for $f H / \beta$, we have

$$
v_{j}(f H / \beta)=\infty
$$

when $0 \leq j \leq n$ and

$$
v_{j}(f H / \beta)=v_{j}(f)-v_{j}(g) \geq 0
$$

when $j \geq n$. So, $f H / \beta \in \operatorname{Int}(E, V)$, completing the proof.

Corollary 5.7. If $E$ is of transcendental type, then $\operatorname{Int}(E, V)$ is a Prüfer domain. 
This handles the situation when $E$ is of transcendental type. When $E$ is of algebraic type, we rely on the next theorem. For an (eventually) pseudo-convergent sequence $\left(a_{i}\right)_{i \in \mathbb{N}}$, we use the notation $\left(v\left(a_{i}\right)\right)_{i \in \mathbb{N}} \rightarrow$ $\infty$ to mean that the pseudo-convergent sequence $\left(a_{i}\right)_{i \in \mathbb{N}}$ is eventually increasing and the values $v\left(a_{i}\right)$ are unbounded.

Theorem 5.8. Consider the following four conditions.

(i) There exists $q \in K[x]$ such that $\left(v_{j}(q)\right)_{j \in \mathbb{N}} \rightarrow \infty$.

(ii) $\operatorname{Br}(E)=(0)$.

(iii) $\operatorname{Int}(E, V)_{\mathfrak{M}_{\infty}}$ is a valuation domain.

(iv) If $q \in K[x]$ and $\left(v_{j}(q)\right)_{j \in \mathbb{N}}$ is eventually increasing, then $\left(v_{j}(q)\right)_{j \in \mathbb{N}}$ $\rightarrow \infty$.

For any pseudo-convergent $E$, we have (i) $\Rightarrow$ (ii) $\Rightarrow$ (iii) $\Rightarrow$ (iv). When $E$ is of algebraic type, (iv) $\Rightarrow$ (i), and hence all four conditions are equivalent.

When $E$ is of algebraic type, it is clear that (iv) $\Rightarrow$ (i). We prove the other implications without any assumption on the type of $E$.

(i) $\Rightarrow$ (ii). Let $q \in K[x]$ be such that $\left(v_{j}(q)\right)_{j \in \mathbb{N}} \rightarrow \infty$. Following [7, Theorem 3], we may assume $q$ is irreducible. Indeed, if $q=q_{1} q_{2}$ for some $q_{1}, q_{2} \in K[x]$, then either $\left(v_{j}\left(q_{1}\right)\right) \rightarrow \infty$ or $\left(v_{j}\left(q_{2}\right)\right) \rightarrow \infty$. So, without loss of generality, assume that $q$ is irreducible.

Let $L$ be the splitting field of $q$ over $K$, and let $w$ be an extension of $v$ to $L$. Factor $q$ as

$$
q(x)=\left(x-\beta_{1}\right)\left(x-\beta_{2}\right) \cdots\left(x-\beta_{t}\right)
$$

for some (not necessarily distinct) $\beta_{\ell} \in L$. Then, for at least one $\ell$, $\left(w\left(\alpha_{j}-\beta_{\ell}\right)\right) \rightarrow \infty$ as $j \rightarrow \infty$. Thus, for sufficiently large $j$,

$$
\begin{aligned}
v\left(\alpha_{j+1}-\alpha_{j}\right) & =w\left(\alpha_{j+1}-\alpha_{j}\right) \\
& =w\left(\left(\alpha_{j+1}-\beta_{\ell}\right)+\left(\beta_{\ell}-\alpha_{j}\right)\right) \\
& =w\left(\beta_{\ell}-\alpha_{j}\right),
\end{aligned}
$$

so $\left(v\left(\alpha_{j+1}-\alpha_{j}\right)\right) \rightarrow \infty$. Hence, $\operatorname{Br}(E)=(0)$.

Before proving (ii) $\Rightarrow$ (iii), we recall a topological definition. A topological space $X$ is precompact when its completion is compact [1, 
Section 4, Definition 2]. Now, [4, Theorem 4.1] asserts that Int $(E, V)$ is a Prüfer domain when $E$ is precompact with respect to the topology on $K$ induced by $v$. So, we will prove that, if $\operatorname{Br}(E)=(0)$, then $E$ is precompact.

(ii) $\Rightarrow$ (iii). Assume that $\operatorname{Br}(E)=(0)$, which implies that $\left(v\left(\alpha_{i+1}-\right.\right.$ $\left.\left.\alpha_{i}\right)\right) \rightarrow \infty$. Also, from [1, Section 3, Proposition 1], it follows that $K$ is metrizable. Let $\widehat{K}$ be the completion of $K$ with respect to $v$, and let $\widehat{E}$ be the corresponding completion of $E$ (that is, the topological closure of $E$ in $\widehat{K}$ ).

The condition $\left(v\left(\alpha_{i+1}-\alpha_{i}\right)\right) \rightarrow \infty$ implies that $\left(\alpha_{i}\right)_{i \in \mathbb{N}}$ is a Cauchy sequence in $K$, so $E$ comprises a convergent sequence in $\widehat{K}$. Hence, every sequence in $\widehat{E}$ has a subsequence converging to a limit point in $\widehat{E}$. Thus, $\widehat{E}$ is compact, $\operatorname{Int}(E, V)$ is a Prüfer domain by $[4$, Theorem 4.1], and so Int $(E, V)_{\mathfrak{M}_{\infty}}$ is a valuation domain.

(iii) $\Rightarrow$ (iv). Here, we prove the contrapositive. Assume $q \in K[x]$ is such that $\left(v_{j}(q)\right)_{j \in \mathbb{N}}$ is eventually increasing, but is bounded above. Let $\beta \in V$ be such that $v_{j}(q)<v(\beta)$ for all $j \in \mathbb{N}$. Let $\phi=\beta / q$. We claim that neither $\phi$ nor $1 / \phi$ is an element of $\operatorname{Int}(E, V)_{\mathfrak{M}_{\infty}}$.

By construction, $v_{j}(1 / \phi)<0$ for all $j$. This violates the conclusion of Lemma 5.3 (ii), so

$$
\frac{1}{\phi} \notin \operatorname{Int}(E, V)_{\mathfrak{M}_{\infty}}
$$

Suppose now that $\phi \in \operatorname{Int}(E, V)_{\mathfrak{M}_{\infty}}$, and write $\phi=f / g$, where $f \in \operatorname{Int}(E, V)$ and

$$
g \in \operatorname{Int}(E, V) \backslash \mathfrak{M}_{\infty}
$$

By Lemma 5.3, $\left(v_{j}(g)\right)_{j \in \mathbb{N}}$ eventually stabilizes at 0 . Hence, for sufficiently large $j$, we have

$$
v_{j}(f)=v_{j}(\phi)=v(\beta)-v_{j}(q) .
$$

But, $\left(v_{j}(q)\right)_{j \in \mathbb{N}}$ is increasing, so $\left(v_{j}(f)\right)_{j \in \mathbb{N}}$ is decreasing. This contradicts Lemma 2.1 (iii). Thus, 


$$
\phi \notin \operatorname{Int}(E, V)_{\mathfrak{M}_{\infty}},
$$

and so Int $(E, V)_{\mathfrak{M}_{\infty}}$ is not a valuation domain.

This completes the proof of Theorem 5.8. The equivalence of (ii) and (iii) when $E$ is algebraic gives us:

Corollary 5.9. Assume that $E$ is of algebraic type. Then, Int $(E, V)$ is Prüfer if and only if $\operatorname{Br}(E)=(0)$.

At this point, we have a complete proof of Theorem 5.2. To summarize the argument: if $E$ is of transcendental type, then $\operatorname{Int}(E, V)$ is Prüfer by Theorem 5.6 and Corollary 5.7. If $\operatorname{Br}(E)=(0)$, then $\operatorname{Int}(E, V)$ is Prüfer by Theorem 5.8. Finally, if $E$ is not of transcendental type and $\operatorname{Br}(E) \neq(0)$, then $E$ must be of algebraic type, and we see that $\operatorname{Int}(E, V)$ is not Prüfer by Corollary 5.9.

It remains to demonstrate that the two conditions in Theorem 5.2, $E$ being of transcendental type and $\operatorname{Br}(E)=(0)$, are, in general, independent of one another. We give two examples to illustrate this.

Example 5.10. Let $\mathbb{Q}^{+}$denote the positive rational numbers. Let $y$ be an indeterminate, let $R=\mathbb{Q}\left[\left\{y^{e}\right\}_{e \in \mathbb{Q}^{+}}\right]$, let $\mathfrak{m}$ be the maximal ideal of $R$ generated by $\left\{y^{e}\right\}_{e \in \mathbb{Q}^{+}}$, and let $V=R_{\mathfrak{m}}$. The fraction field $K$ of $V$ is then a valued field with valuation group isomorphic to the additive group of the rational numbers, and $V$ is a non discrete one-dimensional valuation domain.

Let $E=\left\{y, y^{2}, y^{3}, \ldots\right\}$. Then, $E$ is pseudo-convergent, the breadth of $E$ is $(0)$ and $E$ is of algebraic type because $\left(v_{j}(x)\right) \rightarrow \infty$ as $j \rightarrow \infty$. Note that an alternate example, where the values of $E$ stabilize instead of increasing, is given by

$$
E=\left\{y+y^{2}, y+y^{3}, y+y^{4}, \ldots\right\} .
$$

For this latter choice of $E$, the breadth is still (0), and we have $\left(v_{j}(x-y)\right) \rightarrow \infty$.

Example 5.11. Let $V$ and $K$ be as in Example 5.10. We demonstrate the existence of pseudo-convergent sequences of transcendental type 
and nonzero breadth. (The existence of these sequences was not in doubt prior to this paper, but neither an example nor a proof of existence was found in the available literature.)

It follows from Kaplansky's work in [7] that a pseudo-convergent sequence with a transcendental pseudo-limit is of transcendental type (hence the terminology). So, it suffices to show that there exists a pseudo-convergent sequence in $V$ with a transcendental pseudo-limit and nonzero breadth.

Consider a real number $d=0 . d_{1} d_{2} d_{3} \ldots$, where each $d_{\ell}$ is either 1 or 2. Given such a real number, for each $\ell>0$ let $e_{\ell}=0 . d_{1} d_{2} \ldots d_{\ell}$. For each $i>0$, let

$$
\alpha_{i}=\sum_{\ell=1}^{i} y^{e_{\ell}} .
$$

Take $E_{d}=\left\{\alpha_{1}, \alpha_{2}, \ldots\right\}$. Then,

$$
v\left(\alpha_{i+1}-\alpha_{i}\right)=e_{i+1},
$$

and the sequence $\left(e_{i}\right)_{i>0}$ is increasing, so $E_{d}$ is pseudo-convergent. Also, $\left(e_{i}\right)_{i>0}$ is bounded above, so $\operatorname{Br}\left(E_{d}\right) \neq(0)$. A pseudo-limit of $E_{d}$ in $\widehat{K}$ is given by

$$
L_{d}:=\sum_{\ell=1}^{\infty} y^{e_{\ell}}
$$

Now, for real numbers $d$ and $d^{\prime}$ of the above form, $L_{d^{\prime}}$ is a pseudolimit of $E_{d}$ if and only if $d=d^{\prime}$. Indeed, if $d \neq d^{\prime}$, then $\left(v\left(L_{d^{\prime}}-\alpha_{i}\right)\right)_{i>0}$ will stabilize as soon as the decimal expansions of $d$ and $d^{\prime}$ are different. Since there are uncountably many such $d$, there are uncountably many $L_{d}$. However, $K$ and its algebraic closure are both countable. Hence, there exists a $d$ such that $L_{d}$ is transcendental over $K$, and the corresponding pseudo-convergent sequence $E_{d}$ provides the needed example.

We close with an example, mentioned in the introduction, of an infinite subset $E$ of $V$ that is not precompact, but for which $\operatorname{Int}(E, V)$ is Prüfer. 
Example 5.12. Let $V$ and $K$ be as in the previous two examples. Then, $K$ is metrizable, and we let $\widehat{K}$ be the completion of $K$ with respect to $v$.

Let the $E_{d}$ be as in Example 5.11. Choose $d$ such that $E_{d}$ is of transcendental type; then, $\operatorname{Int}\left(E_{d}, V\right)$ is Prüfer. Note that, for $i>j$, we have

$$
v\left(\alpha_{i}-\alpha_{j}\right)=e_{j+1},
$$

and the sequence $\left(e_{j}\right)_{j>0}$ is bounded above. Because of this, the only Cauchy sequences in $E_{d}$ are those which are eventually constant; hence, $\widehat{E_{d}}=E_{d}$. Moreover, the sequence $\left(\alpha_{1}, \alpha_{2}, \ldots\right)$ has no convergent subsequence, so $\widehat{E_{d}}$ is not compact, and thus $E_{d}$ is not precompact.

Acknowledgments. The authors wish to thank Roswitha Rissner for pointing out-and providing a correction for-an error in a previous version of the proof of Theorem 5.6.

\section{REFERENCES}

1. N. Bourbaki, General topology, Hermann, Paris, 1966 (in English).

2. D. Brizolis, A theorem on ideals in Prüfer rings of integral-valued polynomials, Comm. Alg. 7 (1979), 1065-1077.

3. P.-J. Cahen and J.-L. Chabert, Integer-valued polynomials, Amer. Math. Soc. Surv. Mono. 43, American Mathematical Society, Providence, 1997.

4. P.-J. Cahen, J.-L. Chabert and K.A. Loper, High dimension Prüfer domains of integer-valued polynomials, J. Korean Math. Soc. 38 (2001), 915-935.

5. J.-L. Chabert, Un anneaux de Prüfer, J. Algebra 107 (1987), 1-16.

6. R. Gilmer, Multiplicative ideal theory, Queen's Papers Pure Appl. Math. 90, Kingston, Ontario, Canada, 1992.

7. I. Kaplansky, Maximal fields with valuation, Duke Math. J. 9 (1942), 303-321.

8. K.A. Loper, A classification of all D such that $\operatorname{Int}(D)$ is a Prüfer domain, Proc. Amer. Math. Soc. 126 (1998), 657-660.

9. D.L. McQuillan, On Prüfer domains of polynomials, J. reine angew. Math. 358 (1985), 162-178.

10. _ Rings of integer-valued polynomials determined by finite sets, Proc. Roy. Irish Acad. 85 (1985), 177-184.

11. A. Ostrowski, Untersuchungen zur arithmetischen Theorie der Körper, Math. Z. 39 (1935), 269-404.

12. , Über ganzwertige Polynome in algebraischen Zahlkörpen, J. reine angew. Math. 149 (1919), 117-124. 
13. G. Pólya, Über ganzwertige Polynome in algebraischen Zahlkörpen, J. reine angew. Math. 149 (1919), 97-116.

Department of Mathematics, The Ohio State University-Newark, Newark, $\mathrm{OH} 43055$

Email address: lopera@math.osu.edu

Department of Mathematics, Computer and Information Science, SUNy College at Old Westbury, Old Westbury, Ny 11568

Email address: wernern@oldwestbury.edu 